\title{
Evidence for widespread alterations in cortical microstructure after 32 hours of sleep deprivation
}

\section{Authors:}

Irene Voldsbekk $k^{\mathrm{a}, \mathrm{b}, \mathrm{c},}$, Atle Bjørnerud ${ }^{\mathrm{a}, \mathrm{c}, \mathrm{d}}$, Inge Groote ${ }^{\mathrm{c}}$, Nathalia Zak ${ }^{\mathrm{b}, \mathrm{f}}$, Daniel Roelfs ${ }^{\mathrm{b}, \mathrm{g}}$, Ivan I. Maximov a,b,h, Oliver Geier ${ }^{\mathrm{f}}$, Paulina Due-Tønnessen ${ }^{\mathrm{i}}$, Erlend Bøen', Yvonne S. Kuiper $^{\mathrm{f}}$, Lise-Linn Løkken ${ }^{\mathrm{f}}$, Marie Strømstad ${ }^{\mathrm{a}}$, Taran Y. Blakstvedt ${ }^{\mathrm{a}}$, Bjørn Bjorvatn ${ }^{\mathrm{k}, 1}$, Ulrik F. Maltg , Lars T. Westlye ${ }^{\mathrm{a}, \mathrm{b}, \mathrm{m}}$, Torbjørn Elvsåshagen ${ }^{\mathrm{b}, \mathrm{g}, \mathrm{n}}$, Håkon Grydeland $\mathrm{d}^{\mathrm{a}, \mathrm{o},{ }^{*}}$

${ }^{\text {a }}$ Department of Psychology, University of Oslo, Oslo, Norway.

${ }^{\mathrm{b}}$ Norwegian Centre for Mental Disorders Research (NORMENT), Institute of Clinical Medicine, University of Oslo \& Division of Mental Health and Addiction, Oslo University Hospital, Oslo, Norway.

${ }^{\mathrm{c}}$ Computational Radiology and Artificial Intelligence (CRAI), Division of Radiology and Nuclear Medicine, Oslo University Hospital, Oslo, Norway.

${ }^{\mathrm{d}}$ Department of Physics, University of Oslo, Oslo, Norway.

${ }^{\mathrm{e}}$ Department of Radiology, Vestfold Hospital Trust, Tønsberg, Norway.

${ }^{\mathrm{f}}$ Department of Diagnostic Physics, Division of Radiology and Nuclear Medicine, Oslo University Hospital, Oslo, Norway.

${ }^{\mathrm{g}}$ Institute of Clinical Medicine, University of Oslo, Oslo, Norway.

${ }^{\mathrm{h}}$ Department of Health and Functioning, Western Norway University of Applied Sciences, Bergen, Norway.

${ }^{i}$ Division of Radiology and Nuclear Medicine, Oslo University Hospital, Oslo, Norway.

${ }^{j}$ Psychosomatic and CL Psychiatry, Division of Mental Health and Addiction, Oslo University Hospital, Oslo, Norway.

${ }^{\mathrm{k}}$ Department of Global Public Health and Primary Care, University of Bergen, Bergen, Norway.

${ }^{1}$ Norwegian Competence Center for Sleep Disorders, Haukeland University Hospital, Bergen, Norway.

${ }^{\mathrm{m}}$ KG Jebsen Centre for Neurodevelopmental Disorders, University of Oslo, Oslo, Norway.

${ }^{\mathrm{n}}$ Department of Neurology, Oslo University Hospital, Oslo, Norway.

${ }^{\circ}$ Center for Lifespan Changes in Brain and Cognition, Department of Psychology, University of Oslo, Norway.

* Corresponding authors.

Mail: $\quad$ Department of Psychology, University of Oslo, Postboks 1094, Blindern, 0317 Oslo, Norway.

Phone: $\quad+4741495202$

Email: $\quad$ irene.voldsbekk@psykologi.uio.no

Phone: $\quad+4722845067$

Email: $\quad$ hakon.grydeland@psykologi.uio.no

\section{Keywords:}

Cortical microstructure; $\mathrm{T}_{1} \mathrm{~W} / \mathrm{T}_{2} \mathrm{~W}$ ratio; sleep; sleep deprivation; myelin integrity; wakefulness 


\begin{abstract}
:
Background: Cortical macrostructure is influenced by circadian rhythm and sleep deprivation, yet the microstructural underpinnings of previous human brain MRI findings have remained unclear. The ratio between $T_{1}$-weighted and $T_{2}$-weighted magnetic resonance images $\left(T_{1} w / T_{2} W\right.$ ratio) has been linked to myelin levels and dendrite density, and may offer novel insight into the intracortical microstructure of the sleep deprived brain.
\end{abstract}

Methods: We examined intracortical $\mathrm{T}_{1} \mathrm{w} / \mathrm{T}_{2} \mathrm{~W}$ ratio in 41 healthy young adults (26 women) before and after 32 hours of either sleep deprivation $(n=18)$ or a normal sleep-wake cycle $(n$ $=23)$.

Results: Linear models revealed significant group differences in $\mathrm{T}_{1} \mathrm{w} / \mathrm{T}_{2} \mathrm{~W}$ ratio change after 32 hours in four clusters, including bilateral effects in the insular, cingulate and superior temporal cortex, comprising regions involved in attentional, auditory and pain processing. Across clusters, the sleep deprived group showed an increased $\mathrm{T}_{1 \mathrm{~W}} / \mathrm{T}_{2} \mathrm{~W}$ ratio, while the normal sleepwake group exhibited a reduced ratio. These changes were not explained by in-scanner head movement, and $95 \%$ of the effects across clusters remained significant after adjusting for cortical thickness and hydration.

Conclusions: Compared with a normal sleep-wake cycle, 32 hours of sleep deprivation yields intracortical $\mathrm{T}_{1} \mathrm{~W} / \mathrm{T}_{2} \mathrm{~W}$ ratio increases. While the intracortical changes detected by this study could reflect alterations in myelin or dendritic density, or both, histological analyses are needed to clarify the precise underlying cortical processes. 


\section{Introduction}

Insufficient sleep causes deficits in cognitive and affective processing[1], and is frequently reported among patients with neurodegenerative and psychotic disorders[2]. Chronic sleep deprivation is also common in mood disorders[3]. Paradoxically, acute sleep deprivation has an intriguing antidepressant effect, peaking after around 32 hours[4, 5]. Findings from rodent studies suggest that acute sleep deprivation is linked to structural alterations in the cerebral cortex involving myelin and dendritic spines[6, 7]. However, we lack a clear understanding of the cortical mechanisms underlying sleep deprivation effects in humans.

Using magnetic resonance imaging (MRI) derived indices, several human studies have reported cortical changes following acute sleep deprivation[8,9]. One night without sleep was linked to volume decreases in the insula and parietal cortex[8], and decreased cortical thickness in the medial parietal cortex[9]. However, the interpretation of the underlying mechanisms is limited by the macrostructural nature of these measures. Using microstructural imaging such as diffusion-weighted imaging (DWI), studies have reported sleep deprivation effects on white matter pathways $[10,11]$. This includes alterations in radial diffusivity (RD), which has been related to myelin integrity[12]. DWI has also been applied to measure sleep deprivation effects in the cortex, showing declines in cortical mean diffusivity (MD)[13]. However, the neurobiological correlates of MD changes in the cerebral cortex are complex and not well understood.

Another index of intracortical microstructure is the ratio between $T_{1}$-weighted and $T_{2}-$ weighted images $\left(\mathrm{T}_{1} \mathrm{w} / \mathrm{T}_{2} \mathrm{~W}\right.$ ratio)[14]. Regional variation in $\mathrm{T}_{1} \mathrm{w} / \mathrm{T}_{2} \mathrm{~W}$ ratio has been found to relate to variation in histologically derived myelin levels[14, 15], and, recently, also to dendrite density[16]. While awaiting further histological comparisons, the $\mathrm{T}_{1} \mathrm{w} / \mathrm{T}_{2} \mathrm{~W}$ ratio might be sensitive to the very intracortical properties reported in rodents following sleep deprivation. Interestingly, a recent cross-sectional study reported associations between $\mathrm{T}_{1} \mathrm{w} / \mathrm{T}_{2} \mathrm{~W}$ ratio and 
self-reported sleep quality and sleep duration in several cortical regions[17]. While correlational, these findings may suggest that the $\mathrm{T}_{1} \mathrm{w} / \mathrm{T}_{2} \mathrm{~W}$ ratio is sensitive to sleep-related processes. Hence, testing whether acute sleep deprivation leads to alterations in the $\mathrm{T}_{1} \mathrm{w} / \mathrm{T}_{2} \mathrm{~W}$ ratio might help illuminate the underlying substrates of sleep deprivation effects in humans.

In this study, we investigated the effects of 32 hours of wake and sleep on cortical microstructure using the $\mathrm{T}_{1} \mathrm{w} / \mathrm{T}_{2} \mathrm{~W}$ ratio. In total, 41 healthy young adults underwent MRI before and after either sleep deprivation $(n=18)$ or a normal sleep-wake cycle (NSW, $n=23)$. Based on (i) the links between sleep deprivation and myelin and dendritic spine alterations and (ii) the sensitivity of the $\mathrm{T}_{1} \mathrm{w} / \mathrm{T}_{2} \mathrm{~W}$ ratio to the latter microstructural processes, we hypothesized that the two groups would show significantly different changes in the $\mathrm{T}_{1} \mathrm{w} / \mathrm{T}_{2} \mathrm{~W}$ ratio. To reduce the potential for confounding factors, we monitored a number of Zeitgeber signals, such as food intake, caffeine intake, physical activity and exposure to blue-emitting light. To assess potential functional correlates of cortical changes, we also explored associations between the $\mathrm{T}_{1} \mathrm{w} / \mathrm{T}_{2} \mathrm{~W}$ ratio, and sleepiness and lapses in attention.

\section{Methods and Materials}

\subsection{Ethics statement}

This study was approved by the Regional Committee for Medical and Health Research Ethics, South-Eastern Norway (REK Sør-Øst, ref: 2017/2200) and conducted in line with the Declaration of Helsinki of 2013. All participants gave their written informed consent prior to participation and received NOK 1000.

\subsection{Participants}

The recruitment procedure was described in detail previously[18]. Volunteers were recruited through social media and a national newspaper advert. 127 volunteers underwent clinical 
screening over the phone, and 41 were excluded due to meeting one of the following exclusion criteria: history of any psychiatric disorder, severe or chronic somatic disorder, current intake of any regular medication, smoking, contraindications to MRI or living more than one hour of travel away from the MRI facility. Of the remaining 86 volunteers, 15 withdrew their participation, and 22 were cancelled due to logistic reasons. After study start, one participant was excluded due to illness and two due to claustrophobic reaction in the scanner. An additional five were excluded due to incomplete $\mathrm{T}_{2} \mathrm{~W}$ data. The final sample consisted of 41 healthy adults (mean age $26 \pm 6.9$ years; age range 18 to 46 years; 26 women).

\subsection{Study design}

Figure 1 presents an overview of the study design. Due to time constraints and to reduce the strain on participants, $\mathrm{T}_{2}$-weighted MRI required for $\mathrm{T}_{1} \mathrm{w} / \mathrm{T}_{2} \mathrm{~W}$ ratio estimations were obtained only at the first and last MRI scan. Participants underwent the first MRI scan at time point 1 (TP1) in the morning of the first day at Oslo University Hospital, Rikshospitalet. They arrived fasting after a night of regular sleep in their own home (around 9 AM). After spending the day at the hospital, participants completed their second MRI scan (around 8 PM) and were randomized by draw to either go home to sleep (NSW group) or to stay awake at the hospital during the night (sleep deprivation group). In the morning, the NSW group returned, and both groups underwent their third MRI scan (around 8 AM). They then spent a second day at the hospital. The final scan took place in the afternoon after approximately 32 hours since study start (around 4 PM, TP2).

A blood sample was obtained from each participant after each scan for analysis of hematocrit, to estimate hydration level. Participants followed a standardized activity plan in the company of a research assistant (see Voldsbekk et al., 2020 for a detailed description). To ensure that no one fell asleep during the MRI scans, a camera (Model 12M-i, MRC Systems 
$\mathrm{GmbH}$, Heidelberg, Germany) inside the scanner bore was used to monitor the eyes of the participants. Participants rated their subjective level of sleepiness after each scan and every other hour throughout the study, in addition to completing a computerized test of their alertness every third hour, as outlined below. Participants also filled out questionnaires assessing their health and sleep habits.

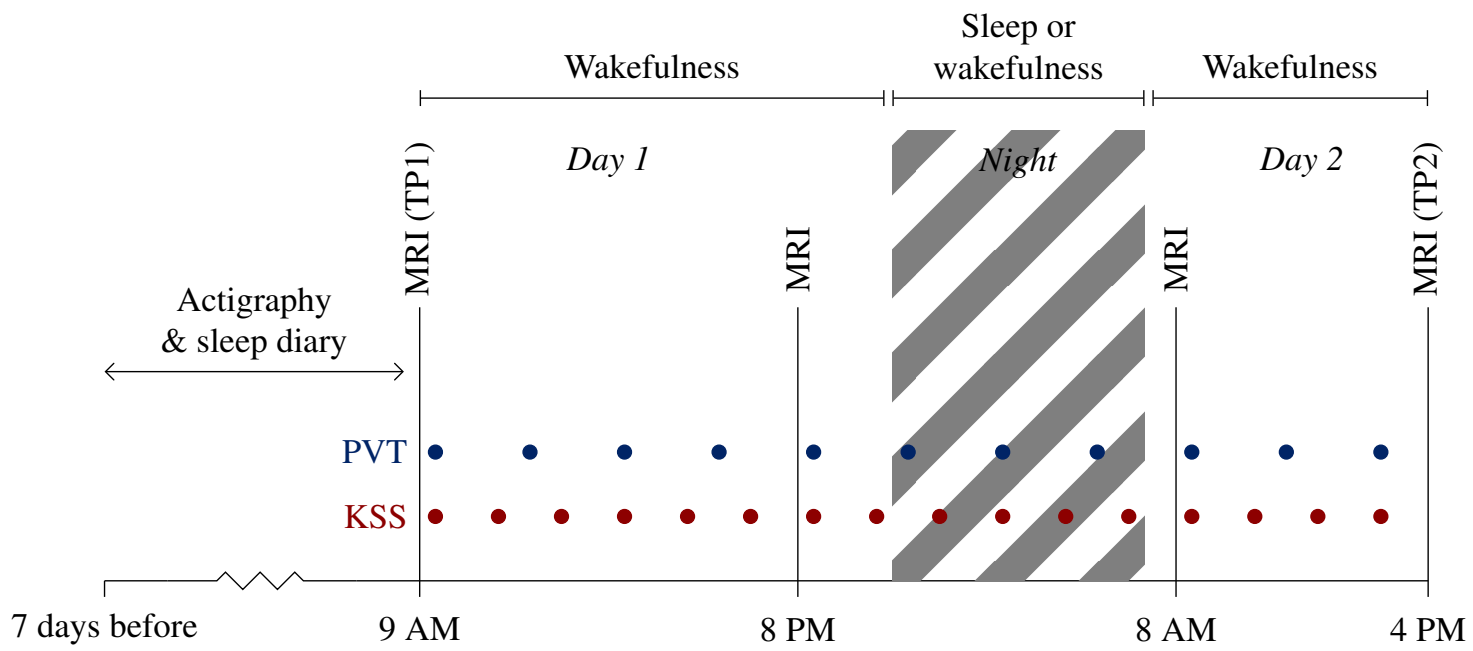

Figure 1. Overview of the study protocol. Participants underwent MRI including $\mathrm{T}_{1} \mathrm{~W}$ - and $\mathrm{T}_{2} \mathrm{~W}$ scans in the morning after a night of sleep (time point (TP)1) and then in the afternoon on the second day (TP2) after 32 hours of either normal sleep-wake or total sleep deprivation. They also underwent MRI (not including $\mathrm{T}_{1} \mathrm{~W}$ - and $\mathrm{T}_{2} \mathrm{~W}$ scans) in the evening on the first day and in the morning on the second day. During the study, participants underwent tests of subjective sleepiness (Karolinska Sleepiness Scale, KSS) and objective alertness (Psychomotor Vigilance Task, PVT) every second and third hour, respectively. Seven days prior to the first MRI scan, participants underwent measurements of sleep habits by actigraphy and self-report sleep diary.

\subsection{Assessment of sleep habits}

Seven days prior to study start, participants recorded their sleep pattern by self-report and actigraphy. Participants in the NSW group also recorded their sleep pattern during the night of the study. Self-report data was recorded each day by a 10-item semi-structured sleep diary[19], which assessed sleep-related behavior and quality. The scale was modified to also include two items on caffeine intake and nicotine intake. Actigraphy data was recorded by a Condor Instruments ActTrust actigraph (São Paulo, Brazil), which measured an individual's 
movements by a digital tri-axial accelerometer with a 60s epoch. In addition, participants completed five standardized questionnaires regarding their sleep habits: the Bergen Insomnia Scale[20], the Epworth Sleepiness Scale[21], the Pittsburgh Sleep Quality Index[22] and the Horne-Østberg Morningness Eveningness Questionnaire[23]. These questionnaires measure insomnia-related symptoms, daytime sleepiness, sleep quality, and chronotype, respectively.

\subsection{Assessment of acute sleepiness and alertness}

During the study, participants completed the Karolinska Sleepiness Scale (KSS), which is a 1item self-report rating of sleepiness on a nine-point Likert scale[24]. To measure objective alertness, participants completed a computerized psychomotor vigilance task (PC-PVT)[25]. For ten minutes, a five-digit millisecond counter was presented as the visual stimulus on a screen with random intervals. Participants clicked a mouse button in response. The task was run using Matlab 2017a (MathWorks, Massachusetts, USA) on a Lenovo laptop V510-15IKB with Windows 10 Pro and a Cooler Master mouse model SGM-1006-KSOA1. The laptop display had a refresh rate of $60 \mathrm{~Hz}$.

\subsection{MRI acquisition}

Imaging was performed on a 3T Siemens Magnetom Prisma scanner (Siemens Healthcare, Erlangen, Germany) using a 32-channel head coil. The scan protocol consisted of a four-echo $\mathrm{T}_{1} \mathrm{~W}$ multi-echo magnetization-prepared rapid gradient-echo sequence (repetition time/echo times $=2530 \mathrm{~ms} / 1.69-3.55-5.41-7.27 \mathrm{~ms}$, field-of-view $=256 \times 256 \mathrm{~mm}^{2}$, voxel size $=$ 1.0x1.0x $1.0 \mathrm{~mm}^{3}$, flip angle $=7^{\circ}$, acceleration factor $=2$ acquisition time $=6 \mathrm{~min}, 3$ seconds) and a bandwidth-matched $\mathrm{T}_{2} \mathrm{~W}$ sequence (repetition time $/$ echo time $=3200 \mathrm{~ms} / 563 \mathrm{~ms}$, field-ofview $=256 \times 256 \mathrm{~mm}^{2}$, voxel size $=1.0 \times 1.0 \times 1.0 \mathrm{~mm}^{3}$, acceleration factor $=2$ acquisition time $=$ 
4 min 24 seconds). The four $\mathrm{T}_{1} \mathrm{w}$ images were used to estimate a root mean square image which was submitted to further analyses[26].

\subsection{MRI preprocessing}

$\mathrm{T}_{1} \mathrm{~W} / \mathrm{T}_{2} \mathrm{~W}$ maps were created using the Human Connectome Project (HCP) processing pipeline (https://github.com/Washington-University/Pipelines)[27], including processing with FreeSurfer version 5.3 (http://surfer.nmr.mgh.harvard.edu), similarly to the processing done by Grydeland and colleagues[28]. The $\mathrm{T}_{1} \mathrm{w}$ volume was divided by the aligned[29] and spline interpolated $\mathrm{T}_{2} \mathrm{~W}$ volume, yielding a $\mathrm{T}_{1} \mathrm{w} / \mathrm{T}_{2} \mathrm{~W}$ ratio volume. For regional $\mathrm{T}_{1} \mathrm{w} / \mathrm{T}_{2} \mathrm{~W}$ maps, a multimodal parcellation was utilized which divided each cerebral hemisphere into 180 regions[30]. $\mathrm{T}_{1} \mathrm{w} / \mathrm{T}_{2} \mathrm{~W}$ values were sampled from the white matter/grey matter boundary, at three cortical depths around the middle of the cortical mantle $(30 \%, 50 \%$, and $70 \%$ into the cortex).

\subsection{Statistical analyses}

In order to ensure homogeneity of the sample, $t$-tests of group differences were run for each demographic and sleep-wake characteristic. To test for group differences in the $T_{1} \mathrm{w} / \mathrm{T}_{2} \mathrm{~W}$ ratio between TP1 and TP2, the symmetrized percentage change (SPC) was calculated, which has been shown to be more robust than percentage change[31]:

$$
\frac{T P 2-T P 1}{T P 2+T P 1} \times 100
$$

Group comparison was then run using linear regression models in R[32] in each of the 360 atlas regions. To reduce potential partial volume effects[33], the main analysis was run using the $\mathrm{T}_{1} \mathrm{~W} / \mathrm{T}_{2} \mathrm{~W}$ ratio measured at a $50 \%$ depth. As a sensitivity analysis to assess the potential effects 
of cortical depth, we then reran the analysis using the $\mathrm{T}_{1} \mathrm{w} / \mathrm{T}_{2} \mathrm{~W}$ ratio from $30 \%$ and $70 \%$ into the cortex. The Euler number, which has been shown to be a useful index of head movement in young adults[34], was extracted from each $\mathrm{T}_{1} \mathrm{w}$ volume and included as a covariate. To control for multiple comparisons, we ran a custom permutation-based cluster size correction analysis across ROIs in R. A cluster was defined as adjacent regions, i.e. regions sharing a border, showing $p$-values below the cluster-forming threshold of $p=.05$. This threshold was chosen to maximize sensitivity in the smaller and less noisy parcellated brain map (compared to a voxelwise map). To build a null model, we then ran 5000 permutations repeating the group analysis, re-shuffling the group membership for each run, and counting the maximum cluster size. Then, the size of the actual clusters was compared with this distribution of 5000 maximum cluster size from random group orderings.

For significant clusters, we extracted the mean $\mathrm{T}_{1} \mathrm{~W} / \mathrm{T}_{2} \mathrm{~W}$ ratio to perform sensitivity analyses. First, we assessed whether the group effect remained significant when including mean cortical thickness across the cluster, and hematocrit, an index of hydration. Second, to explore the functional relevance of changes in $\mathrm{T}_{1} \mathrm{w} / \mathrm{T}_{2} \mathrm{~W}$ ratio from $\mathrm{TP} 1$ to $\mathrm{TP} 2$, interaction analyses were performed between changes in $\mathrm{T}_{1} \mathrm{w} / \mathrm{T}_{2} \mathrm{~W}$ ratio and changes in sleepiness and alertness, as measured by KSS and minor lapses on the PC-PVT. To correct for the multiple tests, the resulting $p$-values were adjusted by applying false discovery rate correction (FDR)[35]. We also utilized neurosynth.org, a data-driven tool for meta-analysis of the large primary literature on task-related functional MRI[36], to identify functions most implicated in the observed changes. The top 25 terms were extracted as a word cloud in which the size and color saturation of the words correspond to the frequencies associated with each term. 


\section{Results}

\subsection{Sleep pattern assessment}

As seen in Table 1, there were no differences in demographics or sleep-wake characteristics between groups. All participants had slept approximately seven hours each night for the past week, as recorded by self-report and corroborated by actigraphy measurements. For the NSW group, there was no significant difference in total sleep time on the night between the first and second day of the study compared to the night prior to study start $(t=-.8, p=.42)$. Across groups, participants slept significantly shorter on the night before the study compared to their weekly average $(t=2.15, p=.03)$.

Table 1. Descriptive statistics for sleep-wake characteristics and tests of differences between sleep deprived and normal sleep-wake groups for each characteristic.

\begin{tabular}{|c|c|c|c|}
\hline & $\begin{array}{l}\text { Sleep-wake } \\
(n=23)\end{array}$ & $\begin{array}{l}\text { Sleep deprived } \\
(n=18)\end{array}$ & $\begin{array}{l}\text { T-test of group } \\
\text { differences }\end{array}$ \\
\hline Age & $25.7(6.97)$ & $26.28(7.05)$ & $-.26(p=.79)$ \\
\hline Sex (no. of women) & $15(65 \%)$ & $11(61 \%)$ & $.26(p=.79)$ \\
\hline PSQI $(0-42)^{\mathrm{a}}$ & $4.5(1.92)$ & $4.78(1.86)$ & $-.46(p=.65)$ \\
\hline MEQ $(16-86)^{\mathrm{a}}$ & $50.52(9.16)$ & $50.11(8.02)$ & $.15(p=.88)$ \\
\hline BIS $(0-42)^{\mathrm{a}}$ & $7.25(5.25)$ & $6.39(4.1)$ & $.58(p=.56)$ \\
\hline $\mathrm{ES}(0-24)^{\mathrm{a}}$ & $6.55(4.48)$ & $5.72(2.63)$ & $.72(p=.47)$ \\
\hline Average TST week before study start (h.hh) ${ }^{1, \mathrm{a}}$ & $7.02(0.93)$ & $6.9(0.94)$ & $.41(p=.68)$ \\
\hline Average TST night before study start (h.hh) ${ }^{1, b}$ & $6.42(0.79)$ & $6.54(1.31)$ & $-.34(p=.74)$ \\
\hline Average TST the night during the study (h.hh) & $6.63(0.78)$ & - & - \\
\hline Change in $\operatorname{KSS}(0-28 \mathrm{~h} \text { into study })^{\mathrm{c}}$ & $-.57(1.5)$ & $2.18(2.96)$ & $-3.48(p=.002)$ \\
\hline Change in PVT $(0-30 \mathrm{~h} \text { into study })^{\mathrm{d}}$ & $1.43(3.34)$ & $3.94(6.45)$ & $-1.4(p=.18)$ \\
\hline \multicolumn{4}{|c|}{$\begin{array}{l}\text { Note. Mean (standard deviation). PSQI; Pittsburgh Global Sleep Quality Index. MEQ; Horne-Östberg } \\
\text { Morningness Eveningness Questionnaire. BIS; Bergen Insomnia Scale. ES; Epworth Sleepiness Scale. } \\
\text { TST; total sleep time. KSS; Karolinska Sleepiness Scale. PVT; psychomotor vigilance test. } \\
{ }^{1} \text { Reported total sleep time as measured by actigraphy and controlled against sleep diary. } \\
{ }^{\text {a }} \text { Missing for one participant. } \\
{ }^{\mathrm{b}} \text { Missing for five participants. } \\
{ }^{\mathrm{c}} \text { Missing for four participants. } \\
{ }^{\mathrm{d}} \text { Missing for eleven participants. }\end{array}$} \\
\hline
\end{tabular}




\subsection{Effect of 32 hours of sleep deprivation and normal sleep-wake on cortical microstructure}

The change from TP1 to TP2 did not differ between groups for hydration or in-scanner head movement $(p=.1$ and $p=.57$, respectively, see Figure S1). As shown in Figure 2A-C, the group comparisons revealed four cortical clusters exhibiting significant differences in $\mathrm{T}_{1} \mathrm{~W} / \mathrm{T}_{2} \mathrm{~W}$ ratio change from TP1 to TP2, spanning 37\% of the ROIs in the right hemisphere (RH) and $16 \%$ in the left hemisphere (LH). Specifically, across clusters, the sleep deprived group showed an increase in $\mathrm{T}_{1} \mathrm{w} / \mathrm{T}_{2} \mathrm{~W}$ ratio, while the NSW group showed a decrease. In the $\mathrm{RH}$, there were 66 regions across two clusters (61and 5 regions, $p<.0001$ and $p=.004$, respectively) in predominantly the insular, cingulate, parietal and superior temporal cortices, while in the LH there were 28 regions across two clusters (13 and 15 regions, $p<.0001$ for both) predominantly in the insular, cingulate, superior temporal and medial frontal cortices. Bilateral effects were observed in the cingulate, insular, and superior temporal cortices.

The first sensitivity analysis showed that the group difference in each cortical cluster remained significant when controlling for hydration and cortical thickness $(p<.05)$, except for the smallest cluster with the initially lowest effect (5-region $\mathrm{RH}$ cluster, $p=.55$ ). To assess whether the results were unduly influenced by one participant in the sleep deprivation group showing high cluster values in three of four clusters (see Figure 2Bi), we excluded this person was and reran the group comparison. As shown in Figure S2, the results were similar to the main analysis, with four clusters, comprising 77 and 14 regions in right and left hemisphere, respectively, showing group differences.

As shown in Figure 3, there was regional variation in the strength of the effect specific to each group . Specifically, the NSW group showed a stronger reduction across medial parietal regions, fusiform gyrus and the posterior cingulate cortex, while the sleep deprived group showed a stronger increase in the anterior cingulate cortex, as well as both lateral and medial 

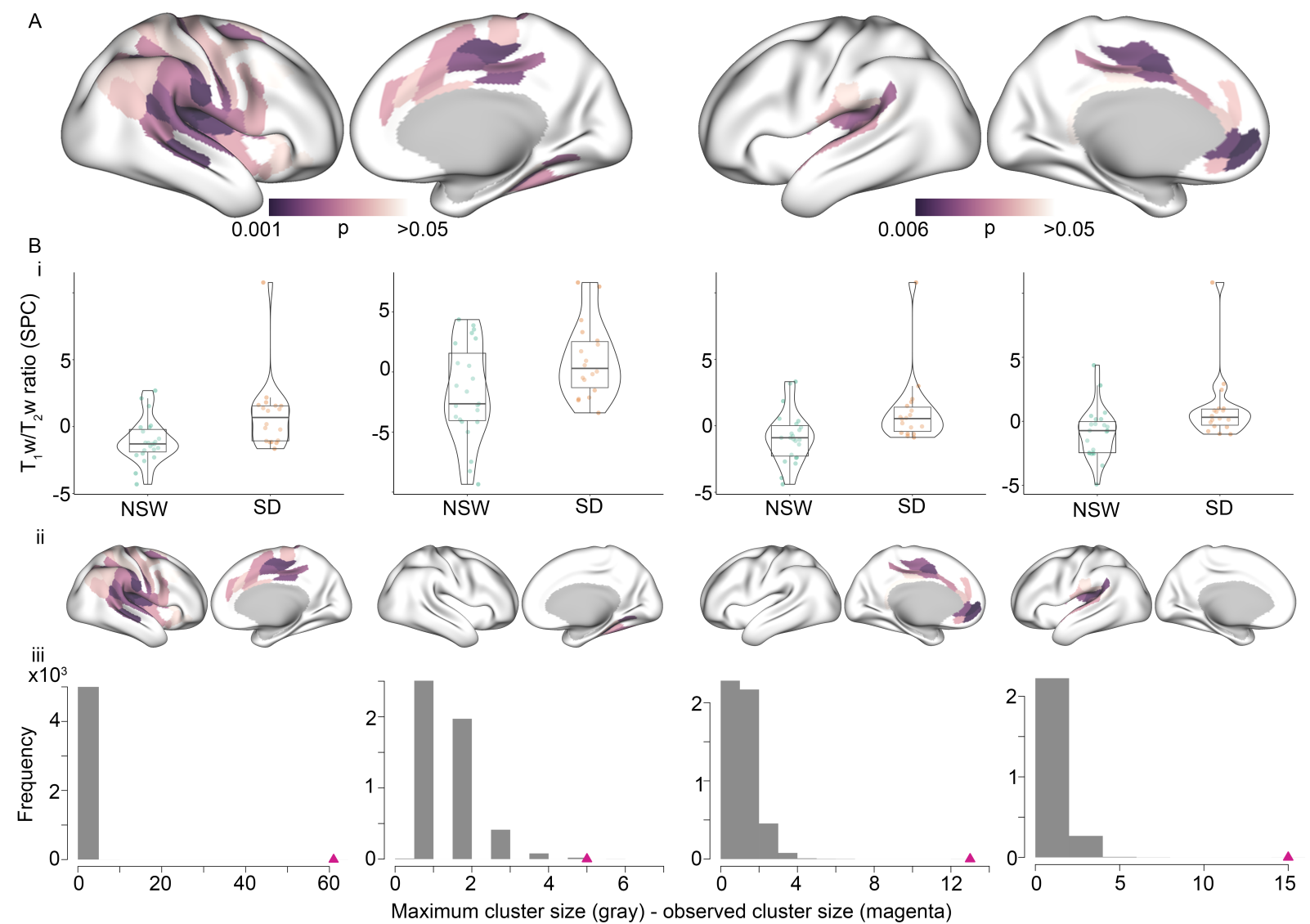

Figure 2. Altered intracortical $\mathrm{T}_{1} \mathrm{w} / \mathrm{T}_{2} \mathrm{~W}$ ratio after 32 hours of either normal sleep-wake (NSW) or sleep deprivation (SD) . A. Surface maps showing the regions of the four clusters showing significant group differences in symmetrized percentage change (SPC) in $\mathrm{T}_{1} \mathrm{w} / \mathrm{T}_{2} \mathrm{~W}$ ratio in the right hemisphere between the SD group and NSW group. Each cluster was $\mathrm{p}<0.05$, and the $p$ values for each region (before cluster-correction) are presented for illustration. Bi. Violin-box-scatter plots for the mean $\mathrm{T}_{1} \mathrm{w} / \mathrm{T}_{2} \mathrm{~W}$ ratio per cluster for participants in each group. Bii. Surface maps of each cluster. Biii. The distribution of maximum cluster sizes across random groupings (grey) with the actual cluster size for each cluster identified with the triangle (magenta).
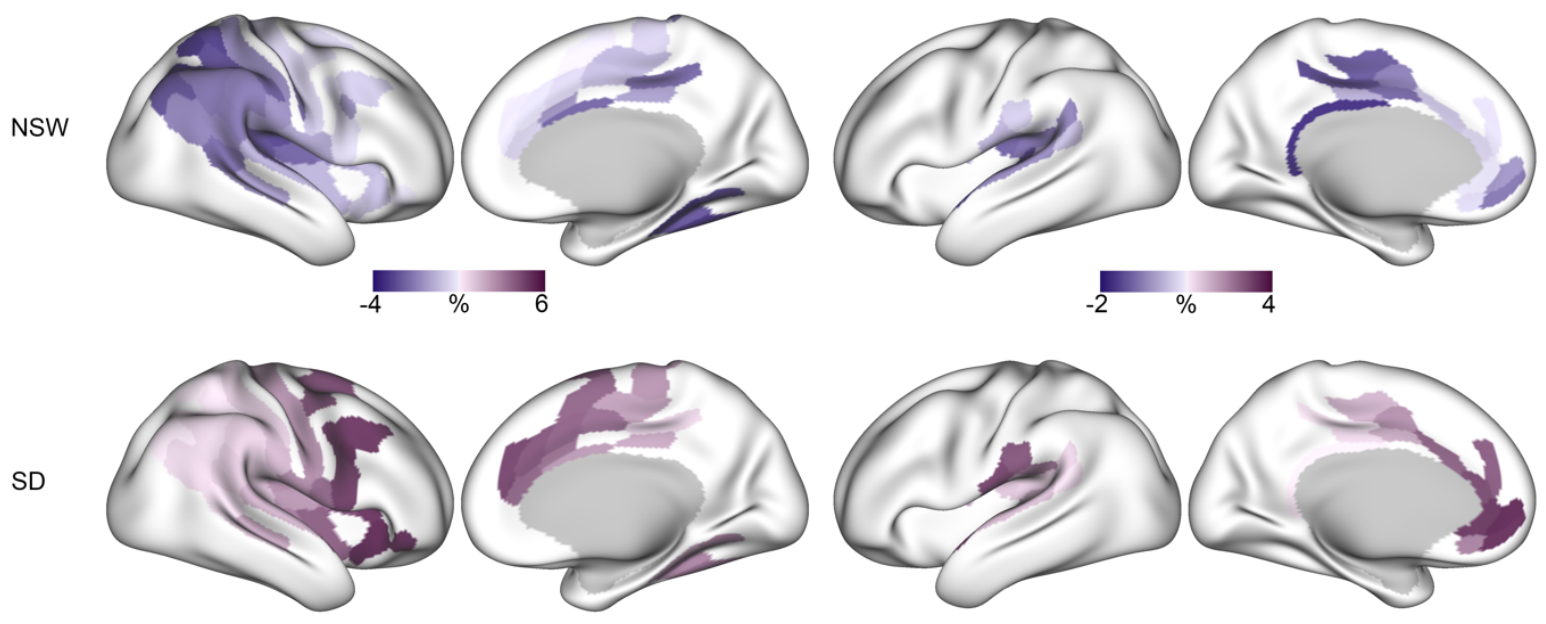

Figure 3. Regional variation in sensitivity to either normal sleep-wake (NSW) or sleep deprivation (SD). Percentage change within each significant cluster for the NSW group (top) and SD group (bottom). 


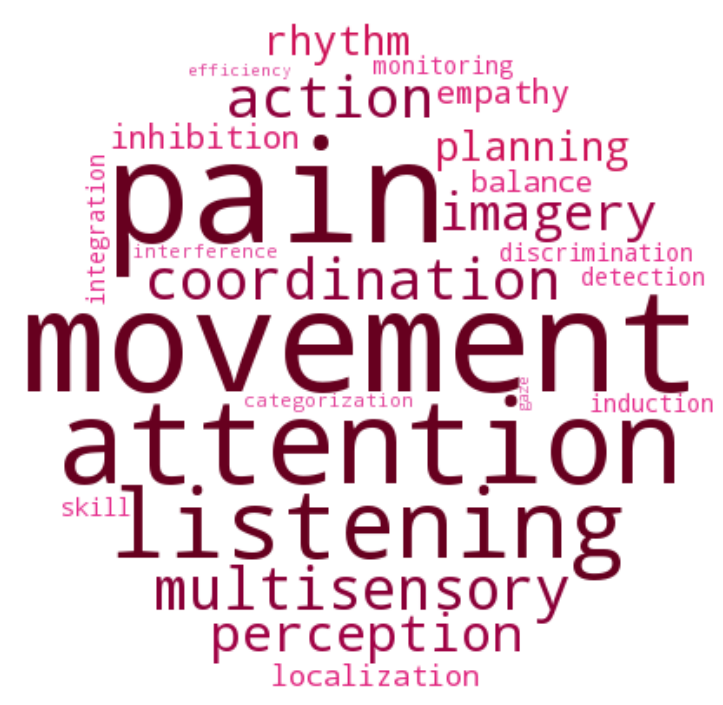

Figure 4. Word cloud based on correlations with NeuroSynth meta-analysis maps across groups. Larger size and color saturation indicate stronger correlation.

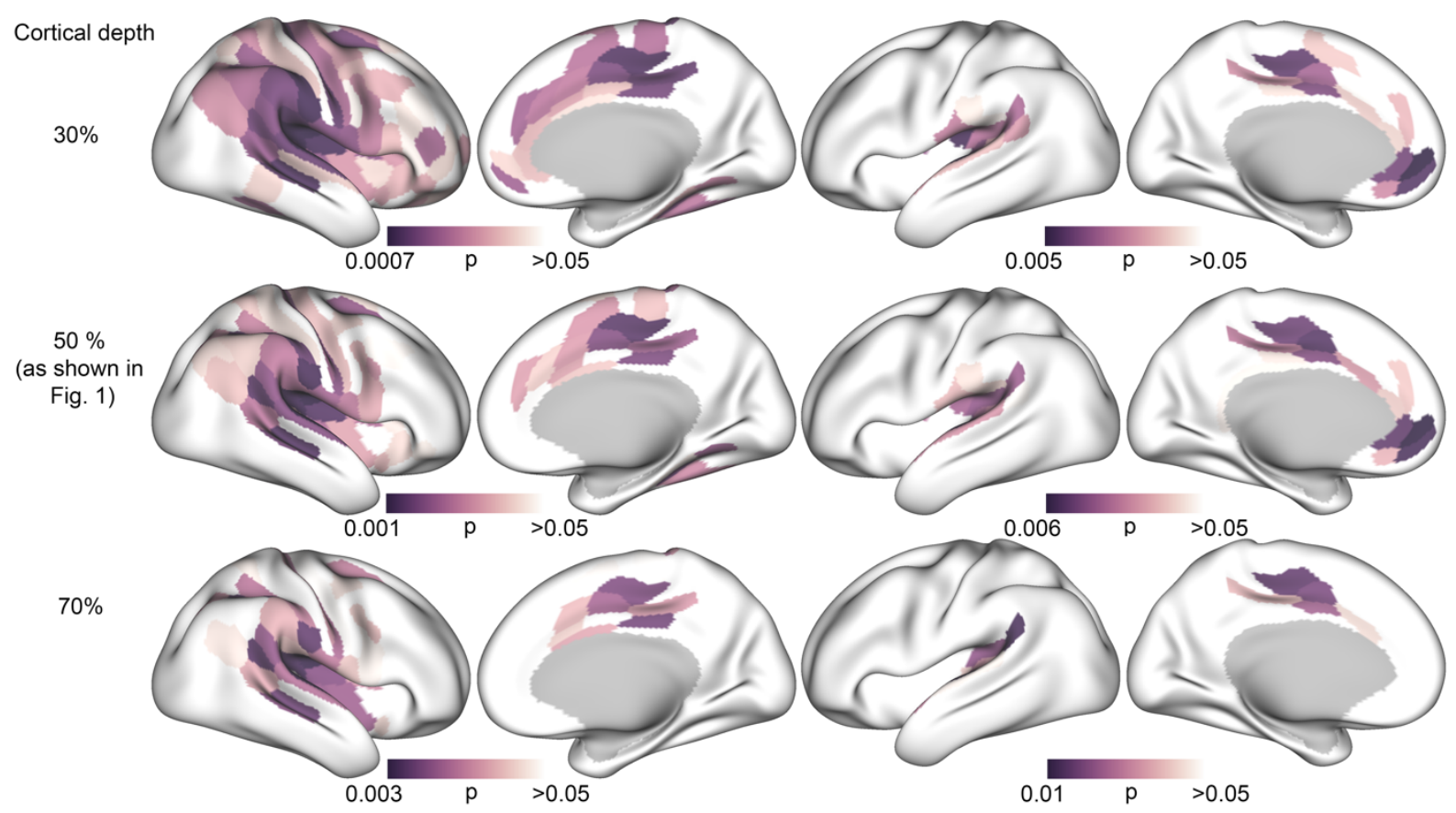

Figure 5. Surface maps showing significant group differences in $\mathrm{T}_{1} \mathrm{w} / \mathrm{T}_{2} \mathrm{~W}$ ratio in the right hemisphere (similar to results reported in Figure $2 \mathrm{~A}$ ) as a function of cortical depth. Top row: $\mathrm{T}_{1} \mathrm{w} / \mathrm{T}_{2} \mathrm{~W}$ ratio sampled from $30 \%$ into the cortex from the white/gray matter boundary. Middle row: $50 \%$ cortical depth (as shown in Figure 2A). Bottom row: 70\% cortical depth.

frontal regions. As determined by neurosynth.org[36] (Figure 4), the observed changes implicated regions most strongly involved in attention, listening, movement and pain. 
To assess whether the higher number of RH regions showing group differences was partly due to a thresholding effect, we summarized the $p$-values of the regions in the LH which were only significant in the RH. Of these $38 \mathrm{LH}$ regions, the median $p$-value was .10 (minimum - maximum is $.02-.55)$, indicating that a portion of the LH regions likely would have been significant with higher statistical power.

The sensitivity analyses assessing the effects of cortical depth, shown in Figure 5, revealed significant group differences using $\mathrm{T}_{1} \mathrm{~W} / \mathrm{T}_{2} \mathrm{~W}$ ratio values extracted from $30 \%$ and $70 \%$ cortical depth. At 30\% depth, a slightly higher number of regions significantly differed between groups (97 RH regions and 29 LH regions), as compared with the results from 50\% depth, while a slightly lower number of significant regions were found at 70\% depth (47 RH regions and 14 LH regions).

\subsection{Associations between changes in cortical microstructure and sleepiness and attention}

The difference in subjective sleepiness and in objectively measured attentional lapses over the 32 hours are presented in Figure S3, and the distribution of change values in Figure S4. The change in subjective sleepiness differed between the groups (see Table 1), with the sleep deprived group showing increased sleepiness (higher KSS at TP2). There was no group difference in change in lapses in attention.

The interaction analyses of an association between $\mathrm{T}_{1} \mathrm{w} / \mathrm{T}_{2} \mathrm{~W}$ ratio changes and sleepiness and attentional lapses, did not show any significant results after FDR-correction $(p$ $>.28$, these analyses were run on the results excluding the participant with a large change value).

The uncorrected results showed a relation between changes in $\mathrm{T}_{1} \mathrm{w} / \mathrm{T}_{2} \mathrm{~W}$ ratio and sleepiness (see Figure S5), with an effect in the 9-region LH cluster $(p=.04)$, while the other clusters did not show effects $(p=.61$ for RH cluster $1, p=.56$ for $\mathrm{RH}$ cluster 2 , and $p=.25$ for 
LH cluster). The effect was due to a negative correlation between $\mathrm{T}_{1} \mathrm{w} / \mathrm{T}_{2} \mathrm{~W}$ change and sleepiness change in the sleep deprived group $(r=-.7, p=.003)$, while the NSW group showed an opposite, and weaker relation $(r=.27, p=.27)$. Thus, in the sleep deprivation group, a stronger increase in $\mathrm{T}_{1} \mathrm{w} / \mathrm{T}_{2} \mathrm{~W}$ ratio was related to lower subjective sleepiness.

The uncorrected results of changes in $\mathrm{T}_{1} \mathrm{w} / \mathrm{T}_{2} \mathrm{~W}$ ratio and lapses in attention (see Figure S6) showed a weak trend of an interaction in the 4-region RH cluster $(p=.092)$, while the other clusters did not show effects ( $p=.51$ for RH cluster $1, p=.16$ for LH cluster 1 , and $p=.5$ for LH cluster 2). This trend was due to a positive correlation between $\mathrm{T}_{1} \mathrm{w} / \mathrm{T}_{2} \mathrm{~W}$ change and alertness change in the NSW group $(r=.67, p=.012)$, while the sleep deprivation group showed an opposite, and weaker relation $(r=-.16, p=.54)$.

\section{Discussion}

The findings of the present study indicate that 32 hours of sleep deprivation yields different intracortical changes in the $\mathrm{T}_{1} \mathrm{~W} / \mathrm{T}_{2} \mathrm{~W}$ ratio than compared to an NSW cycle. The differences between the sleep deprivation and the NSW groups were more prominent in the RH, but were observed in both hemispheres in insular, cingulate and superior temporal cortices and included regions involved in attentional, auditory, movement and pain processing[36]. Across regions, the sleep deprived group showed an increased $\mathrm{T}_{1} \mathrm{w} / \mathrm{T}_{2} \mathrm{~W}$ ratio, whereas the NSW group exhibited a reduced ratio. The effects were not explained by an estimate of in-scanner head movement, were present at various depths in the cortex, and $95 \%$ of the effects remained significant after adjusting for cortical thickness and hydration. Although speculative, candidate neurobiological mechanisms of the observed cortical effects include myelin and dendrite density, previously linked to the $\mathrm{T}_{1} \mathrm{w} / \mathrm{T}_{2} \mathrm{~W}$ ratio[14-16].

We found group differences in $\mathrm{T}_{1} \mathrm{w} / \mathrm{T}_{2} \mathrm{~W}$ ratio change in the bilateral cingulate, insula, and superior temporal cortices and in right parietal and left middle frontal regions. Although 
this is the first study of $\mathrm{T}_{1} \mathrm{w} / \mathrm{T}_{2} \mathrm{~W}$ ratio in sleep deprivation, these results overlap with findings from three previous studies. The first study reported reduced cortical volume mainly in the insular, parietal, posterior cingulate, motor, and somatosensory cortices after 36 hours of sleep deprivation[8]. The second study found increased grey matter density in the frontal pole and the superior and middle frontal gyri, as well as decreased volume and thickness in the temporal pole after 24 hours of sleep deprivation[37]. The third study reported reduced thickness in bilateral medial parietal regions, yet did not detect a significant group by time interaction effect when compared to a NSW group[9]. Thus, the results of the present and previous studies suggest that acute sleep deprivation is associated with cortical alterations, mainly in frontal, temporal, parietal, and insular cortices. The current study points to new effects in the bilateral anterior cingulate, the superior temporal (including auditory cortex), and the left medial frontal regions. This difference in results may reflect the differences in study designs, but also indicate that the microstructural $\mathrm{T}_{1} \mathrm{w} / \mathrm{T}_{2} \mathrm{~W}$ ratio has greater sensitivity to the intracortical effects of wake and sleep deprivation.

We observed increased $\mathrm{T}_{1} \mathrm{w} / \mathrm{T}_{2} \mathrm{~W}$ ratio in the sleep deprived group. Further histological studies are needed to elucidate the neurobiology underlying these $\mathrm{T}_{1} \mathrm{w} / \mathrm{T}_{2} \mathrm{~W}$ ratio changes, yet they may be linked to wake-related processes, circadian rhythm mechanisms, or a combination of the two[38]. Although the precise neural effects of wake and sleep remain to be clarified, the synaptic homeostasis hypothesis posits that wake and sleep are associated with net increases and decreases, respectively, in densities of synapses and dendrites[39]. Consistent with this hypothesis, spontaneous and enforced wake in animals were linked to increased dendritic branching and synapse number[6, 40, 41] and reduced synapse pruning[42]. Thus, although speculative, $\mathrm{T}_{1} \mathrm{~W} / \mathrm{T}_{2} \mathrm{~W}$ ratio changes within the sleep deprivation group of the present study could be related to wake-induced increases in synaptic and dendritic densities. 
An alternative explanation for the increased $\mathrm{T}_{1} \mathrm{w} / \mathrm{T}_{2} \mathrm{~W}$ ratio after sleep deprivation is cortical myelin changes. The $\mathrm{T}_{1} \mathrm{w} / \mathrm{T}_{2} \mathrm{~W}$ ratio correlates with cortical myelin content[14], and the ratio was higher in myelinated than demyelinated cortex in multiple sclerosis[15]. There is a scarcity of studies of cortical myelin after sleep deprivation in humans, yet two previous MRI studies reported RD reductions in human white matter after extended wake[10, 18]. Reduced RD is, in general, associated with increased myelination[12]. Furthermore, a growing body of animal data links sleep and wake to myelin and oligodendrocyte alterations[7, 43]. Thus, further studies should clarify whether changes to myelin and oligodendrocytes contribute to the $\mathrm{T}_{1} \mathrm{w} / \mathrm{T}_{2} \mathrm{~W}$ ratio increases found in the current study.

We observed a $\mathrm{T}_{1} \mathrm{w} / \mathrm{T}_{2} \mathrm{~W}$ ratio reduction within the NSW group from the morning of the first study day to the afternoon of the second day. We speculate that $\mathrm{T}_{1} \mathrm{~W} / \mathrm{T}_{2} \mathrm{~W}$ changes from morning to evening of the first study day would have been reversed by the night of sleep, and that the NSW group changes detected here reflect morning- to-afternoon processes of the second study day. These hypotheses must be tested in future studies since the current work obtained $\mathrm{T}_{2}$-weighted MRI only at baseline (TP1) and after 32 hours (TP2). Notwithstanding this limitation, the $T_{1} \mathrm{w} / \mathrm{T}_{2} \mathrm{~W}$ ratio reduction within the NSW group is consistent with recent studies reporting time-of-day effects on other MRI modalities[9, 10, 13, 18, 44-49].

Taken together, the directionality of the $\mathrm{T}_{1} \mathrm{w} / \mathrm{T}_{2} \mathrm{~W}$ ratio changes in the two groups were, interestingly, in the opposite direction. Simultaneous MRI and histological analyses are required to clarify whether regular wake length, e.g., from morning to the same afternoon, could be associated with neural processes that qualitatively differ from those induced by sleep deprivation. The relationships between wake length and cortical alterations are not necessarily linear. In support of this notion, one study observed decreases in cortical thickness from morning to afternoon (2 hours)[46], whereas another study detected increased cortical thickness from morning to evening (14 hours)[9]. Furthermore, the $\mathrm{T}_{1} \mathrm{~W} / \mathrm{T}_{2} \mathrm{~W}$ ratio changes observed in 
the current study could also reflect the likely complex and dynamic relationship between wakeinduced neural processes and the circadian rhythm of the cortex. The current understanding of sleep entails two separable, yet interacting processes, namely the homeostatic process, in which sleep pressure accumulates as a function of time spent awake, and the circadian process, which oscillates at about 24-hour periods and is regulated by exposure to daylight[38].

The $\mathrm{T}_{1} \mathrm{w} / \mathrm{T}_{2} \mathrm{~W}$ ratio group differences comprised regions involved in attentional processes, which are amongst the first to deteriorate in response to sleep deprivation[50]. However, we found no significant relationship between attentional lapses and $\mathrm{T}_{1} \mathrm{w} / \mathrm{T}_{2} \mathrm{~W}$ ratio. Previous studies reported significant associations between sleepiness and changes in cortical thickness[9] and cortical density[37] after sleep deprivation. Consistent with these results, we found a weak association between reported sleepiness and $\mathrm{T}_{1} \mathrm{w} / \mathrm{T}_{2} \mathrm{~W}$ ratio change in one of the smaller clusters, but this did not survive FDR-correction. Thus, while the group differences suggest significant effects of sleep deprivation, the functional consequences of the intracortical $\mathrm{T}_{1} \mathrm{~W} / \mathrm{T}_{2} \mathrm{~W}$ ratio changes among the sleep deprived individuals remain to be elucidated.

Several study limitations should be noted. First, participants had slept less the night before the study than their weekly average. However, if we assume that sleep reverses microstructural changes after wakefulness, we expect less sleep to result in attenuation, rather than inflation, of any sleep deprivation-related changes. Second, participants in the NSW group went home to sleep, which was measured with an actigraph and a sleep diary. Sleep in a laboratory with polysomnography would have resulted in greater control of exposure to Zeitgebers and sleep length. Third, the study did not include $\mathrm{T}_{2} \mathrm{~W}$ MRI acquisitions from the evening of the first study day and the next morning, which limited our ability to interpret the temporal dynamics of the $\mathrm{T}_{1} \mathrm{w} / \mathrm{T}_{2} \mathrm{~W}$ ratio changes. Finally, the sample size was modest, and the analyses indicated that more LH effects would have been significant with higher statistical 
power. The lateralization of $\mathrm{T}_{1} \mathrm{w} / \mathrm{T}_{2} \mathrm{~W}$ ratio effects with more significant clusters in the $\mathrm{RH}$ should therefore be cautiously considered.

The current study provides evidence that compared with a NSW cycle, 32 hours of sleep deprivation yields intracortical microstructural changes as indexed by the $\mathrm{T}_{1} \mathrm{~W} / \mathrm{T}_{2} \mathrm{~W}$ ratio. These effects were observed within regions linked to attentional, auditory, movement and pain processing and were not explained by an estimate of in-scanner head movement, minimally affected by cortical thickness, and hydration, and were present at various depths in the cortex. The $\mathrm{T}_{1} \mathrm{w} / \mathrm{T}_{2} \mathrm{~W}$ ratio changes could reflect alterations in myelin or dendrite density, or both, and histological analyses are needed to clarify the precise underlying cortical processes. 


\section{Acknowledgements:}

This project was funded by researchs grant from the Norwegian South-East Health

Authorities (2018077, 2017090, 2015078), the Research Council of Norway (249795), the

Centre for Digital Life Norway, the Ebbe Frøland foundation, the Norwegian Competence

Center for Sleep Disorders, Haukeland University Hospital, Bergen (www.sovno.no), the

University of Oslo Life Science summer scholarship for students and a research grant from Mrs. Throne-Holst. 
bioRxiv preprint doi: https://doi.org/10.1101/2021.06.22.449439; this version posted June 22, 2021. The copyright holder for this preprint

(which was not certified by peer review) is the author/funder, who has granted bioRxiv a license to display the preprint in perpetuity. It is made available under aCC-BY-NC 4.0 International license.

\section{Disclosures:}

T.E. received speaker's honoraria from Lundbeck and Janssen Cilag and is a consultant to

BrainWaveBank and Synovion. N.Z. received speaker's honoraria from Lundbeck. 


\section{References}

1. Killgore, W.D., Effects of sleep deprivation on cognition. Progress in brain research, 2010. 185: p. 105-129.

2. Wulff, K., et al., Sleep and circadian rhythm disruption in psychiatric and neurodegenerative disease. Nat Rev Neurosci, 2010. 11(8): p. 589-99.

3. Peterson, M.J. and R.M. Benca, Sleep in mood disorders. Psychiatric Clinics, 2006. 29(4): p. 1009-1032.

4. Boland, E.M., et al., Meta-Analysis of the Antidepressant Effects of Acute Sleep Deprivation. Journal of Clinical Psychiatry, 2017. 78(8): p. e1020-e1034.

5. $\mathrm{Wu}, \mathrm{C}$. and E. Bunney, The biological basis of an antidepressant response to sleep deprivation and relapse: Review and hypothesis. 1990(January): p. 14-21.

6. de Vivo, L., et al., Ultrastructural evidence for synaptic scaling across the wake/sleep cycle. Science, 2017. 355(6324): p. 507-510.

7. Bellesi, M., et al., Effects of Sleep and Wake on Oligodendrocytes and Their Precursors. Journal of Neuroscience, 2013. 33(36): p. 14288-14300.

8. Dai, X.J., et al., Plasticity and Susceptibility of Brain Morphometry Alterations to Insufficient Sleep. Front Psychiatry, 2018. 9: p. 266.

9. Elvsåshagen, T., et al., Evidence for cortical structural plasticity in humans after a day of waking and sleep deprivation. NeuroImage, 2017. 156(May 2016): p. 214-223.

10. $\quad$ Elvsåshagen, T., et al., Widespread changes in white matter microstructure after a day of waking and sleep deprivation. PLoS ONE, 2015. 10(5): p. 1-15.

11. Voldsbekk, I., et al., Sleep and sleep deprivation differentially alter white matter microstructure: A mixed model design utilising advanced diffusion modelling. NeuroImage, 2021. 226: p. 117540. 
12. Song, S.K., et al., Dysmyelination revealed through MRI as increased radial (but unchanged axial) diffusion of water. NeuroImage, 2002. 17(3): p. 1429-1436.

13. Bernardi, G., et al., Sleep reverts changes in human gray and white matter caused by wake-dependent training. NeuroImage, 2016. 129: p. 367-377.

14. Glasser, M.F. and D.C. Van Essen, Mapping Human Cortical Areas $<e m>I n$ Vivo <lem $>$ Based on Myelin Content as Revealed by T1- and T2-Weighted MRI. The Journal of Neuroscience, 2011. 31(32): p. 11597-11616.

15. Nakamura, K., et al., T1-/T2-weighted ratio differs in demyelinated cortex in multiple sclerosis. Annals of Neurology, 2017. 82(4): p. 635-639.

16. Righart, R., et al., Cortical pathology in multiple sclerosis detected by the T1/T2weighted ratio from routine magnetic resonance imaging. Ann Neurol, 2017. 82(4): p. 519-529.

17. Toschi, N., L. Passamonti, and M. Bellesi, Sleep quality relates to emotional reactivity via intracortical myelination. Sleep, 2020.

18. Voldsbekk, I., et al., Evidence for wakefulness-related changes to extracellular space in human brain white matter from diffusion-weighted MRI. NeuroImage, 2020. 212: p. $116682-116682$.

19. Bjorvatn, B., Søvndagbok. 2018.

20. Pallesen, S., et al., A new scale for measuring insomnia: the bergen insomnia scale'. Perceptual and motor Skills, 2008: p. 691-706.

21. Johns, M.W., A New Method for Measuring Daytime Sleepiness: The Epworth Sleepiness Scale. Sleep, 1991. 14(6): p. 540-545.

22. Buysse, D.J., et al., The Pittsburgh Sleep Quality Index: a new instrument for psychiatric practice and research. Psychiatry research, 1989. 28(2): p. 193-213. 
23. Horne, J.A. and O. Östberg, A Self Assessment Questionnaire to Determine Morningness Eveningness in Human Circadian Rhythms. International Journal of Chronobiology, 1976. 4(2): p. 97-110.

24. Åkerstedt, T. and M. Gillberg, Subjective and objective sleepiness in the active individual. International Journal of Neuroscience, 1990. 52(1-2): p. 29-37.

25. Khitrov, M.Y., et al., PC-PVT: A platform for psychomotor vigilance task testing, analysis, and prediction. Behavior Research Methods, 2014. 46(1): p. 140-147.

26. Han, X., et al., Reliability of MRI-derived measurements of human cerebral cortical thickness: the effects of field strength, scanner upgrade and manufacturer.

Neuroimage, 2006. 32(1): p. 180-194.

27. Glasser, M.F., et al., The minimal preprocessing pipelines for the Human Connectome Project. Neuroimage, 2013. 80: p. 105-124.

28. Grydeland, H., et al., Waves of Maturation and Senescence in Micro-structural MRI Markers of Human Cortical Myelination over the Lifespan. Cerebral Cortex, 2019. 29(3): p. 1369-1381.

29. Greve, D.N. and B. Fischl, Accurate and robust brain image alignment using boundary-based registration. NeuroImage, 2009. 48(1): p. 63-72.

30. Glasser, M.F., et al., A multi-modal parcellation of human cerebral cortex. Nature, 2016. 536(7615): p. 171-178.

31. Berry, D.A. and G.D. Ayers, Symmetrized percent change for treatment comparisons. The American Statistician, 2006. 60(1): p. 27-31.

32. Team, R.C., R: A language and environment for statistical computing. 2018: Vienna, Austria.

33. Huntenburg, J.M., P.-L. Bazin, and D.S. Margulies, Large-Scale Gradients in Human Cortical Organization. Trends in Cognitive Sciences, 2018. 22(1): p. 21-31. 
34. Rosen, A.F., et al., Quantitative assessment of structural image quality. Neuroimage, 2018. 169: p. 407-418.

35. Benjamini, Y. and Y. Hochberg, Controlling the False Discovery Rate: A Practical and Powerful Approach to Multiple Testing. Journal of the Royal Statistical Society: Series B (Methodological), 1995. 57(1): p. 289-300.

36. Yarkoni, T., et al., Large-scale automated synthesis of human functional neuroimaging data. Nature methods, 2011. 8(8): p. 665-670.

37. Sun, J., et al., Alteration of Brain Gray Matter Density After 24 h of Sleep Deprivation in Healthy Adults. Front Neurosci, 2020. 14: p. 754.

38. Borbély, A.A., et al., The two-process model of sleep regulation: A reappraisal. Journal of Sleep Research, 2016. 25(2): p. 131-143.

39. Tononi, G. and C. Cirelli, Sleep and the Price of Plasticity: From Synaptic and Cellular Homeostasis to Memory Consolidation and Integration. Neuron, 2014. 81(1): p. 12-34.

40. Bushey, D., G. Tononi, and C. Cirelli, Sleep and Synaptic Homeostasis: Structural Evidence in Drosophila. Science, 2011. 332(6037): p. 1576-1581.

41. Donlea, J.M., M.N. Alam, and R. Szymusiak, Neuronal substrates of sleep homeostasis; lessons from flies, rats and mice. Current Opinion in Neurobiology, 2017. 44: p. 228-235.

42. Li, W., et al., REM sleep selectively prunes and maintains new synapses in development and learning. Nat Neurosci, 2017. 20(3): p. 427-437.

43. Bellesi, M., et al., Myelin modifications after chronic sleep loss in adolescent mice. Sleep, 2018. 41(5).

44. Elvsåshagen, T., et al., Cerebral blood flow changes after a day of wake, sleep, and sleep deprivation. NeuroImage, 2019. 186: p. 497-509. 
45. Kaufmann, T., et al., The brain functional connectome is robustly altered by lack of sleep. NeuroImage, 2016. 127: p. 324-332.

46. Trefler, A., et al., Impact of time-of-day on brain morphometric measures derived from T1-weighted magnetic resonance imaging. NeuroImage, 2016. 133: p. 41-52.

47. Thomas, C., et al., Impact of time-of-day on diffusivity measures of brain tissue derived from diffusion tensor imaging. NeuroImage, 2018. 173: p. 25-34.

48. Hodkinson, D.J., et al., Circadian and Homeostatic Modulation of Functional Connectivity and Regional Cerebral Blood Flow in Humans under Normal Entrained Conditions. Journal of Cerebral Blood Flow \& Metabolism, 2014. 34(9): p. 14931499.

49. Jiang, C., et al., Diurnal Variations in Neural Activity of Healthy Human Brain Decoded with Resting-State Blood Oxygen Level Dependent fMRI. Frontiers in Human Neuroscience, 2016. 10: p. 634-634.

50. Goel, N., et al., Neurocognitive consequences of sleep deprivation. Seminars in neurology, 2009. 29(4): p. 320-339. 